\title{
A comparison of hydrodynamic and analytic predicted blast pressure profiles
}

\author{
G. M. Stunzenas \& E. L. Baker \\ US Army Armament Research Development and Engineering Center, \\ USA
}

\begin{abstract}
Modeling the structural response to blast relies on accurate descriptions of the blast loading pressure profiles. Traditionally, empirically based blast pressure histories are used for this modeling. However, the structural response and geometric configuration can strongly affect the blast loading profile, particularly for close-in blast loading configurations. As a result, high rate continuum modeling is being increasingly applied to directly resolve both the blast profiles and structural response. A variety of computer models exist for the purpose of analyzing blast pressures associated with different types of explosive charges and ranges. These computer models range from simple empirically based analytic models based off of the Hopkinson cube root scaling to multi-physics high rate finite element approaches, commonly known as "hydrocodes", which are capable of tracking shocks through the conservation equations of continuum mechanics. The purpose of this paper is to provide comparisons of blast profiles predicted by analytic models with a hydrodynamic model at various standoffs. Three computer models; BlastX, Conwep, and ALE3D, were used to model the detonation of five pounds of TNT. Pressure profiles for various standoffs were gathered from each computer model and compared. The ALE3D result is greatly dependant on the mesh size and appears to converge to the BlastX and Conwep solutions with increased mesh resolution.
\end{abstract}

Keywords: blast, explosives, modelling.

\section{Introduction}

Modeling the structural response to blast relies on accurate descriptions of the blast loading pressure profiles. Traditionally, empirically based blast pressure 
histories are used for this modeling. However, the structural response and geometric configuration can strongly affect the blast loading profile, particularly for close-in blast loading configurations [1-3]. As a result, high rate continuum modeling is being increasingly applied to directly resolve both the blast profiles and structural response. An explosion produces shock waves in air, which extend outward from the point of detonation. This shock wave is composed of a highly nonlinear shock front, which decays as the distance from the source increases. This nonlinearity is characterized by a sharp, instantaneous increase in pressure, called the peak incident overpressure. The velocity of the shock is supersonic in the medium in which it travels. The gas molecules behind the shock travel at a lower particle velocity and generally make up what is referred to as the shock wind. As the volume in which the shock travels increases, the peak pressures associated with the shock decrease [2]. A variety of computer models exist for the purpose of analyzing blast pressures associated with different types of explosive charges and ranges. These computer models range from simple empirically based analytic models based off of the Hopkinson cube root scaling to multi-physics high rate finite element approaches, commonly known as "hydrocodes", which are capable of tracking shocks through the conservation equations of continuum mechanics. The purpose of this paper is to provide comparisons of blast wave profiles in open air predicted by analytic models with a high rate continuum model at various standoffs. Open air blast profiles were predicted using two analytic models: Conwep, BlastX, and a high rate continuum model: ALE3D. A comparison of the peak incident overpressure was made for a 5 pound spherical TNT charge at various distances from the charge.

\section{Analytic blast modeling}

Conwep blast calculations are conducted using a set of empirically based equations and curves. These empirically based scaled blast curves have been generated for spherical and hemispherical TNT charges [2]. These curves are used to predict blast variables including time of arrival, impulse, peak incident and peak reflected pressures. These curves were created based on Hopkinson cube root scaling [3], which relates the characteristic properties of the blast wave from an explosion of one energy level to that of another. According to this scaling law, the pressure at a certain distance from the charge is proportional to the cube root of the energy yield. Figure 1 presents scaled blast relationship curves [2] and is representative of how Conwep computes peak blast pressures and other blast characteristics for an open air detonation using a spherical TNT charge.

A scaled distance is calculated, which is the ratio of the distance from the charge to the cube root of the explosive weight. This scaled distance is then used to compute all of the variables associated with the particular blast wave of interest. Table 1 shows the peak incident overpressure computed by Conwep at various distances from the charge. This table also shows the results of using the chart alone with the scaled distance calculations. Based on the similar results, 


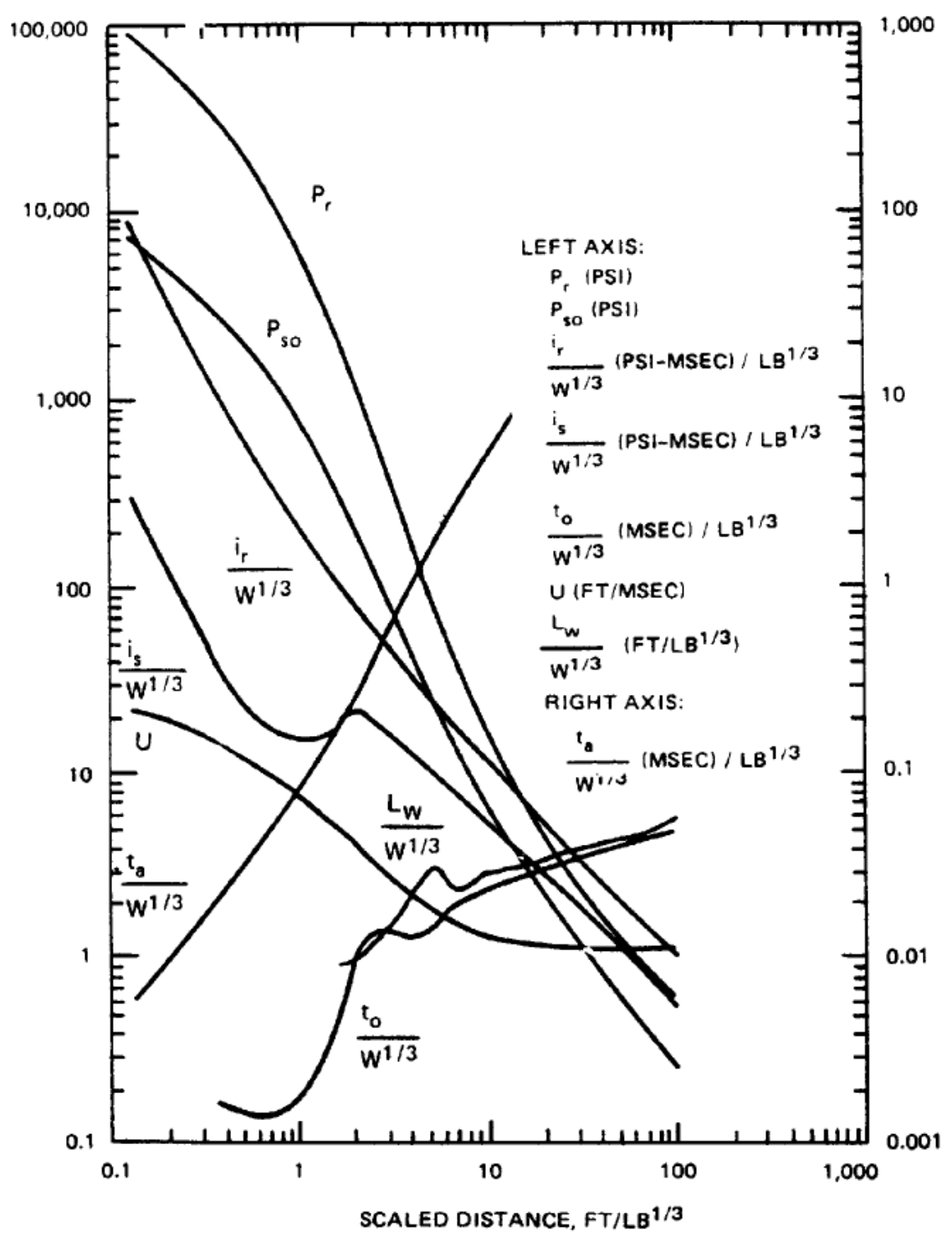

Figure 1: Blast wave characteristics vs. scaled distance for spherical TNT charge.

this demonstrates how Conwep uses this particular curve and scaling law to calculate air blast properties at various ranges. 
One thing worth noting is that Figure 1 is on a log-log scale, which tends to makes accurate values difficult to assess from the graphical representations. This is likely the cause of any discrepancy between using the chart alone and the Conwep results. BlastX treats shock wave effects with a ray-based semiempirical model. Similar to Conwep, it uses tabular blast data for spherical and cylindrical explosive charges. The blast data tables are based on hydrocode calculations for a $1 \mathrm{~kg}$ charge (of various explosives) at a standard set of atmospheric conditions. Results for other charge weights and atmospheric conditions are obtained similar to Conwep using Hopkinson scaling, as discussed above. BlastX uses the tabular values to calculate wave forms by interpolation of blast pressures, particle velocity, and density that were computed using the $1 \mathrm{~kg}$ spherical charge $[5,6]$.

Table 1: Comparison between peak pressures obtained from Conwep and Fig. 1.

\begin{tabular}{|c|c|c|}
\hline $\begin{array}{c}\text { Distance from } \\
\text { Charge (ft) }\end{array}$ & $\begin{array}{c}\text { Conwep Pressure } \\
\text { Results (PSI) }\end{array}$ & $\begin{array}{c}\text { Using Chart and Scaled } \\
\text { Distance (PSI) }\end{array}$ \\
\hline 2.5 & 425.7 & 410 \\
\hline 3 & 295.5 & 298 \\
\hline 3.5 & 213.8 & 200 \\
\hline 4 & 160 & 150 \\
\hline 4.5 & 123.2 & 110 \\
\hline 5 & 97.17 & 95 \\
\hline 5.5 & 78.26 & 80 \\
\hline 6 & 64.18 & 68 \\
\hline 6.5 & 53.46 & 60 \\
\hline
\end{tabular}

\section{High rate continuum blast modeling}

ALE3D is an arbitrary Lagrangian/Eulerian high rate finite difference hydrocode which is used to model fluid and solid elastic-plastic response of materials. A mesh is used to define a volume in space, and the conservation equations (mass, momentum, and energy) of continuum mechanics are applied and integrated through time, giving an updated nodal response to different forces, pressures, stresses, and strains [4]. The TNT charge was modeled using a standard JonesWilkins-Lee (JWL) detonation products equation of state. The air was modeled using a constant gamma equation of state. Table 2 presents the explosive products and air equations of state parameters. 
Table 2: $\quad$ TNT JWL parameters and air constant gamma parameters used for the ALE-3D calculations.

\begin{tabular}{|c|c|c|c|c|c|}
\hline & $\begin{array}{c}\text { Density } \\
(\mathrm{g} / \mathrm{cc})\end{array}$ & Gamma & $\begin{array}{c}\text { CJ Pressure } \\
(\mathrm{GPa})\end{array}$ & $\begin{array}{c}\text { Det Velocity } \\
(\mathrm{cm} / \text { microsecond) }\end{array}$ & EOS Coefficients \\
\hline $\begin{array}{c}\text { TNT JWL } \\
\text { Parameters }\end{array}$ & 1.63 & 2.66 & 17.7 & 689 & $\begin{array}{c}\mathrm{A}=3.712 \mathrm{Mbar} \\
\mathrm{B}=.03231 \mathrm{Mbar} \\
\mathrm{R} 1=4.15 \\
\mathrm{R} 2=.95 \\
\text { Omega }=.30\end{array}$ \\
\hline $\begin{array}{c}\text { Air } \\
\text { Constant } \\
\text { Gamma } \\
\text { Parameters }\end{array}$ & .000129 & 1.4 & N/A & N/A & N/A \\
\hline
\end{tabular}

\section{Results}

Conwep, BlastX, and ALE3D were used to compute the peak pressure profiles of a $5 \mathrm{lb}$ spherical charge of TNT, detonated in open air. The distance from the charge was varied, and the results are summarized in the Table 3. Figure 2 shows some images that were captured from the ALE3D simulation for the open airblast. Certain parameters were modified in ALE3D, while holding the mesh resolution constant to examine affect on the produced blast profiles and discrepancy between the different model outputs. The mesh resolution used for this study was approximately .12 cells $/ \mathrm{mm}$, resulting in 5.8 million total cells.
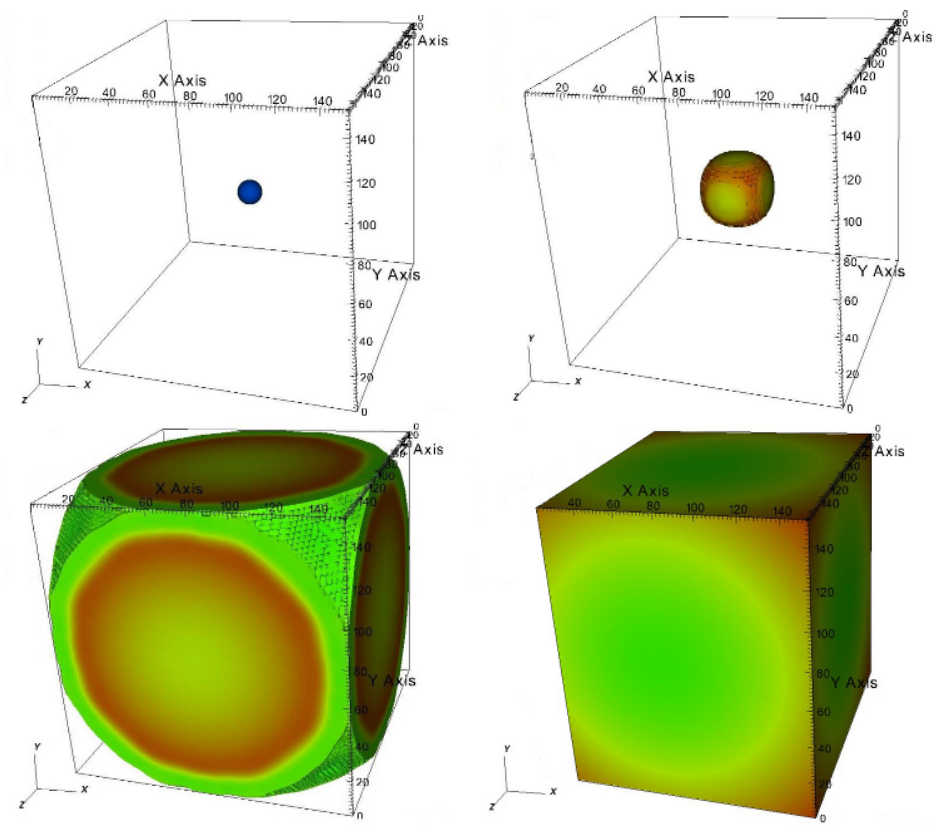

Figure 2: $\quad$ ALE3D open airblast simulation images - Blast front position. 
The initial purpose of this comparison was to study how the kinetic energy advection method affected the results. Since this term is quadratic in nature, rather than linear, energy can sometimes be lost if the velocity varies strongly in a calculation. The kinetic energy advection method can be set so that the energy lost computationally during integration for a shock is added to the internal energy (fracke $=1$ ). There has been some debate about what this does to the resulting calculation, as the objective is to keep a strong shock without putting the material on a wrong adiabat [4]. ALE3D was run in Eulerian mode for the purposes of this comparison. Table 3 summarizes the results for BlastX, Conwep, and ALE3D at various distances with the kinetic energy advection term (fracke) set to 0 and 1 . The information in Figure 3 depicts the results in this table:

Table 3: $\quad$ Comparison of peak pressures for Conwep, BlastX, and ALE3D.

\begin{tabular}{|c|c|c|c|c|}
\hline $\begin{array}{c}\text { Distance } \\
\text { from charge } \\
\text { (ft) }\end{array}$ & $\begin{array}{c}\text { Conwep } \\
\text { Pressure } \\
\text { (psi) }\end{array}$ & $\begin{array}{c}\text { BlastX } \\
\text { Pressure } \\
\text { (psi) }\end{array}$ & $\begin{array}{c}\text { ALE3D - } \\
\text { Eulerian } \\
\text { (fracke = 1) } \\
\text { Pressure (psi) }\end{array}$ & $\begin{array}{c}\text { ALE3D - } \\
\text { Eulerian } \\
\text { (fracke = 0) } \\
\text { Pressure (psi) }\end{array}$ \\
\hline 2.5 & 425.7 & 400.3 & 226 & 220 \\
\hline 3 & 295.5 & 278.4 & 175 & 171 \\
\hline 3.5 & 213.8 & 212.8 & 142 & 144 \\
\hline 4 & 160 & 161.5 & 119 & 121 \\
\hline 4.5 & 123.2 & 123.6 & 99.4 & 103 \\
\hline 5 & 97.17 & 96.57 & 90.2 & 86.1 \\
\hline 5.5 & 78.26 & 76.78 & 82 & 82.6 \\
\hline 6 & 64.18 & 62.7 & 73 & 77.5 \\
\hline 6.5 & 53.46 & 52.25 & 69.7 & 69.9 \\
\hline
\end{tabular}

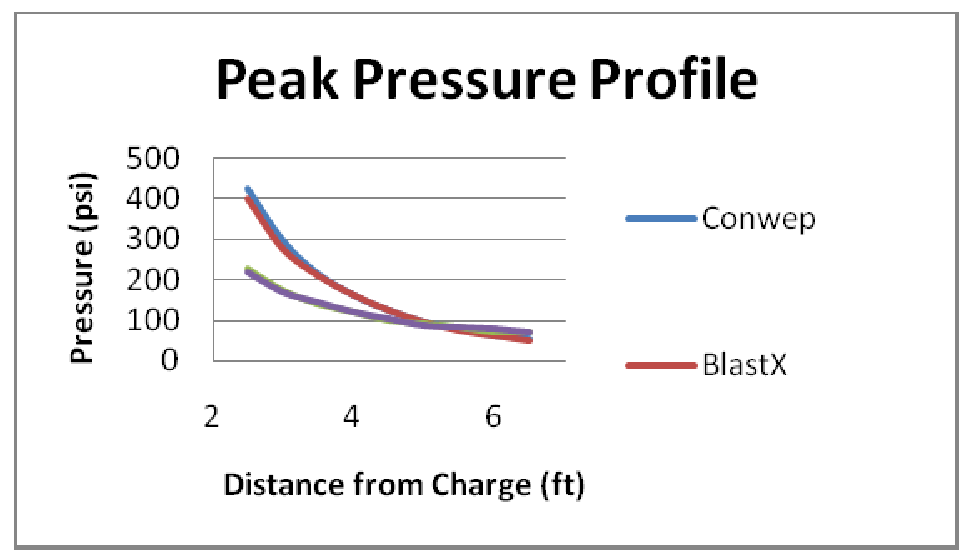

Figure 3: Graphical comparison of peak pressures between Conwep, BlastX, and ALE3D at various standoffs. 
It can be seen that the kinetic energy advection term did not have a significant effect on the calculations, but the results using ALE3D were much different than the empirically-based Conwep and BlastX. However, the Conwep and BlastX calculations produce similar blast results. In order to further investigate the differences between the high rate continuum modeling and the analytic blast models, several further modifications were made to the ALE3D computations. In an attempt to better track the shock wave propagation, monotonic artificial viscosity was used, rather than the default linear-quadrate rate dependant artificial viscosity. Subsequently, different mesh ratios were used to allow the shock to expand as it travelled away from the charge. This approach was investigated, as it is well known that large changes in mesh size affect shock propagation calculations. Finally, the resolution of the calculation was investigated, by increasing the number of elements to determine dependency on mesh size. Table 4 summarizes the results of the analysis at a distance of 2.5 feet from the charge. The results indicate that convergence to the analytic results appears to be occurring with increased mesh resolution.

Table 4: Peak pressure results using ALE3D with modified input parameters.

\begin{tabular}{|c|c|c|}
\hline $\begin{array}{c}2.5 \text { Feet From } \\
\text { Charge }\end{array}$ & $\begin{array}{c}\text { Analysis } \\
\text { Description }\end{array}$ & $\begin{array}{c}\text { Resulting Pressure } \\
\text { (psi) }\end{array}$ \\
\hline Run 1 & $\begin{array}{c}\text { Eulerian, q } \\
\text { weighting, slight } \\
\text { increase in mesh } \\
\text { size }\end{array}$ & 260 \\
\hline Run 2 & $\begin{array}{c}\text { q weighting } \\
\text { Modequipotential } \\
\text { rather than Eulerian }\end{array}$ & 262 \\
\hline Run 3 & $\begin{array}{c}\text { Eulerian, q } \\
\text { weighting, different } \\
\text { mesh ratios }\end{array}$ & 281 \\
\hline Run 4 & $\begin{array}{c}\text { Eulerian, q } \\
\text { weighting, different } \\
\text { mesh ratios }\end{array}$ & 284 \\
\hline Run 5 & $\begin{array}{c}\text { Eulerian, q } \\
\text { weighting, finer } \\
\text { mesh }\end{array}$ & 300 \\
\hline Run 6 & $\begin{array}{c}\text { Eulerian, } \mathrm{q} \\
\text { weighting, finer } \\
\text { mesh } \\
\end{array}$ & 326 \\
\hline Run 7 & 10 million elements & 335 \\
\hline Run 8 & 18 million elements & 339 \\
\hline
\end{tabular}




\section{Conclusion}

More investigation is required if the true discrepancy between these computer models is to be determined, but based on the above analysis, it is quite evident that tracking shocks in ALE3D is greatly dependant on the mesh size. For the 5 LBS charge investigated, it appears that a mesh resolution of at least .15 cells $/ \mathrm{mm}$ is required. The artificial viscosity term also had some impact on the calculations. Conwep and BlastX can not solely be depended upon when conducting blast analyses on structures simply due to the fact that they are empirical in nature. Much of the data calculated is based on curves and interpolated from existing databases. In the future, hydrodynamic codes, such as ALE3D will become increasingly necessary to solve these highly nonlinear and dynamic problems due to their capability of resolving both shock and structural response at the same time.

\section{References}

[1] Ngo, T., Mendis, P., Gupta, A. \& Ramsay, J., Blast Loading and Blast Effects on Structures - An Overview, EJSE Special Issue: Loading on Structures, pp. 76 - 91, 2007.

[2] Headquarters, Department of the Army, Fundamentals of Protective Design for Conventional Weapons, Technical Manual TM 5-855-1, 3 November, 1986.

[3] Headquarters, Department of the Army, Engineering Design Handbook, Explosions in Air, AMC Pamphlet AMCP 706-181, 15 July 1974.

[4] Lawrence Livermore National Laboratories, ALE3D High Performance Multi-Physics Simulations, LLNL-MI-413853.

[5] Commission on Engineering and Technical Systems (CETS), Protecting Buildings from Bomb Damage: Transfer of Blast-Effects Mitigation Technologies from Military to Civilian Applications, National Academy Press: Washington, D.C., 1995.

[6] Ray J.C., Armstrong, B.J. \& Slawson, T.R., Airblast Environment Beneath a Bridge Overpass, Journal of the Transportation Research Board of the National Academies, vol. 1827, pp. 63 - 68, 2003. 